\title{
Anomalous diamagnetic susceptibility in 13-atom Platinum
}

\section{nanocluster superatoms**}

\author{
E. Roduner*, C. Jensen, J. van Slageren, R. A. Rakoczy, O. Larlus, and M. Hunger
}

Abstract: We are used to predict diamagnetic susceptibilities $\chi_{\mathrm{D}}$ to a good approximation by atomic increments since there is normally little dependence on the chemical environment. Surprisingly, we find from SQUID magnetization measurements that $\chi_{\mathrm{D}}$ per Pt atom of zeolite supported $\mathrm{Pt}_{13}$ nanoclusters exceeds that of $\mathrm{Pt}^{2+}$ ions by a factor of 37-50. The observation verifies an earlier theoretical prediction. The phenomenon is understood nearly quantitatively on the basis of a simple expression for diamagnetic susceptibility and the superatom nature of the 13-atom near-spherical cluster. The two main contributions come from ring currents in the delocalized hydride shell, the second one from cluster molecular orbitals hosting the Pt $5 \mathrm{~d}$ and 6 s electrons.

$N_{\text {anosize particles of metallic elements have attracted great }}$ attention since their properties often deviate markedly from those of the bulk metal. For example, micro- and nanosize platinum powder has been reported to become superconducting, ${ }^{[1-3]}$ while bulk Pt does not. There are predictions that metal clusters with $10^{2}-10^{3}$ free carriers may become superconducting. ${ }^{[4]}$ Furthermore, at low temperature, Pt nanoparticles and clusters show pronounced superparamagnetism, ${ }^{[5-7]}$ whereas bulk $\mathrm{Pt}$ has a small positive magnetic susceptibility due to Pauli paramagnetism that is slightly temperature dependent. ${ }^{[8]}$ Other work predicts a greatly enhanced diamagnetism in metal clusters, ${ }^{[9,10]}$ but there has been no experimental verification of this. Diamagnetism is an important indicator of superconductivity as well, which raises the interest in this property for small clusters even further. However, since the London penetration depth of superconductors is normally on the order of $100 \mathrm{~nm}$ one should not expect to find an ideal diamagnetism with full exclusion of the magnetic field for objects of less than $1 \mathrm{~nm}$ diameter. Moreover, since magnetic properties reflect the wavefunction they are of general interest in physics as well as in chemistry.

We have previously demonstrated that monodisperse icosahedral or cuboctahedral platinum nanoclusters with $13 \pm 2$ atoms can be prepared supported within the porous structure of $\mathrm{NaY}$ zeolite. $^{[6]}$ Although they are all of the same size they exist in three different magnetic states: $15-20 \%$ in a high-spin state with a magnetic moment of $\mu=3.7 \pm 0.4$ and $3.0 \pm 0.4 \mu_{\mathrm{B}}$ for $\mathrm{Pt}_{13}$ and $\mathrm{Pt}_{13} \mathrm{H}_{\mathrm{m}}$, respectively, a very small fraction of $<1 \%$ contributes to the spin- $-1 / 2$ EPR signal, and the rest is diamagnetic. ${ }^{[7]}$ The coexistence of different electronic states of the same cluster may be rationalized by different local environments, such as the presence of different numbers of zeolite framework $\mathrm{Al}$ atoms or extra framework cations,
$\mathrm{Na}^{+}$or $\mathrm{K}^{+}$, in close proximity to the cluster, and the extent of hydrogen coverage will also play a role.

Here we resume the subject of magnetism with these clusters using a specially synthesized iron-free L zeolite (Supporting Information) to avoid any interference of iron and ambiguities in the interpretation of the data. The potassium form of the zeolite was exchanged using a $3 \mathrm{mM}$ solution of $\left[\mathrm{Pt}\left(\mathrm{NH}_{3}\right)_{4}\right] \mathrm{Cl}_{2}$. The product was washed, dried and then calcined in a flux of $\mathrm{O}_{2}$ while the sample was heated at a rate of $0.5 \mathrm{~K} \mathrm{~min}^{-1}$, and keeping the final temperature $(623 \mathrm{~K})$ for $5 \mathrm{~h}$. Reduction was then performed in flowing $\mathrm{H}_{2}$ at a heating rate of $4 \mathrm{~K} \mathrm{~min}^{-1}$ and keeping the sample at $503 \mathrm{~K}$ for $1 \mathrm{~h}$. The structure of the obtained zeolite sample was characterized by XRD and solid-state NMR (see Supporting Information, Figures S1 and S2), while the investigation of the Pt clusters was performed by $\mathrm{EXAFS}^{[6,11]}$ and EPR spectroscopy. Details about the experimental procedure which are crucial for a defined cluster formation are found elsewhere. ${ }^{[12,13]}$ The extent of $\mathrm{H}_{2}$ or $\mathrm{D}_{2}$ coverage was determined by sequential addition of calibrated hydrogen aliquots and monitoring the pressure. ${ }^{[11]}$

The EPR spectrum of a $\mathrm{Pt}_{13} \mathrm{D}_{\mathrm{X}}$ cluster shows a multiplet spectrum with a regular splitting (see Figure S3). The pattern fits well the hyperfine interaction of the unpaired electron with 12 equivalent Pt nuclei in natural isotopic abundance and suggests a superatom structure (Supporting Information). The resolved multiplet is clear evidence for a molecular rather than metallic character of the cluster.

Magnetization measurements were performed with a $\mathrm{Pt}_{13} \mathrm{H}_{38}$ and a $\mathrm{Pt}_{13} \mathrm{H}_{18}$ sample using a superconducting quantum interference device (SQUID, quantum design MPMXL7). Paramagnetism was discussed in detail in Ref. 12. In short, Curie behavior with a CurieWeiss constant $\theta=0$ was observed in a temperature range of 20-70 K. A magnetic moment of 0.236(2) $\mu_{\mathrm{B}}$ per Pt atom was obtained for both samples, ${ }^{[13]}$ corresponding to $J \approx 1.5$ for the 13 -atom cluster or 3 unpaired electrons if the moment is due to electron moments only. These values oscillate significantly with the amount of chemisorbed hydrogen, and they show some development with time. ${ }^{[12,13]}$ Previous XMCD measurements of two similar samples with not quantitatively known hydrogen coverage revealed a ratio of orbital to spin magnetic moments, $m_{L} / m_{S}$ of $0.30(2)$, and thus to an orbital angular momentum contribution to paramagnetism of ca. $23 \%$, significantly less than for bulk Pt (28\%). ${ }^{[7]}$

The magnetization saturation curves shown in Figure 1(A) reveal the paramagnetic nature of the samples due to a fraction of high spin clusters at low temperature. All magnetizations are corrected for the contribution of the plain zeolite. At room 
temperature and fields $>0.2 \mathrm{~T}$, however, the magnetizations turn negative, scaling linearly with field, so the behavior is clearly dominated by diamagnetism.

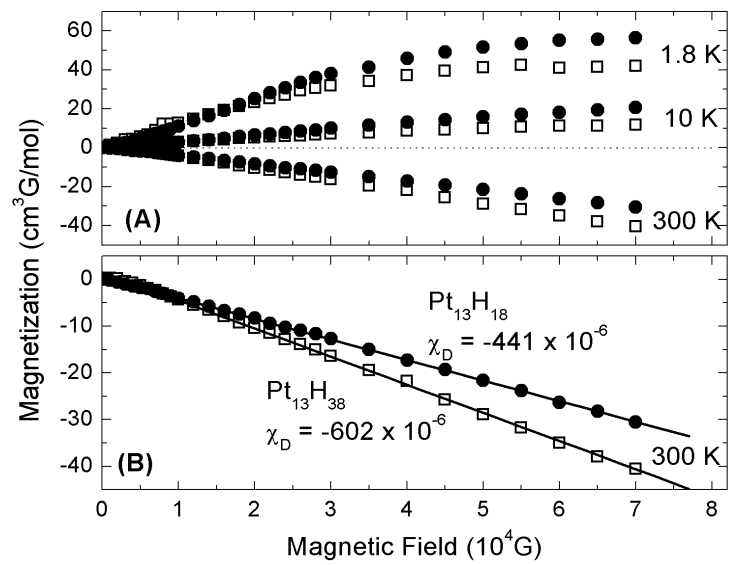

Figure 1. SQUID magnetization measurements obtained with 5.5 wt.\% $\mathrm{Pt}$ supported on iron-free KL-zeolite for $\mathrm{Pt}_{13} \mathrm{H}_{18}$ (full dots) and $\mathrm{Pt}_{13} \mathrm{H}_{38}$ (empty squares) (A) and for $300 \mathrm{~K}$ on an expanded scale (B). The magnetization of the same amount of zeolite in the absence of $\mathrm{Pt}$ was subtracted. The numbers are given per mol Pt atoms

Above $\sim 40 \mathrm{~K}$, the EPR signal diminishes much more quickly than predicted by the Curie law, indicating that also some chemical equilibrium is involved. ${ }^{[14]}$ If this hold also for the high spin state then it is not surprising that nearly no influence of the paramagnetic fraction is observed at $300 \mathrm{~K}$. Elsewhere, it was demonstrated for the 13-atom clusters $\mathrm{Mn@Sn_{12 }}$ that excitation of Jahn-Teller active vibrations has a pronounced effect on the spin structure and causes rapid transition between states. ${ }^{[15]}$ Evidence for the excitation of vibrations at low frequencies as expected for the heavy atom $\mathrm{Pt}$ clusters comes from the relaxation rate of the $\mathrm{Pt}_{13} \mathrm{D}_{\mathrm{X}}$ EPR signal which increases with the square of temperature. ${ }^{[14]}$

The raw data of the field-dependent magnetization measurements are given in Figure S4, together with an explanation of the subtraction of the zeolite contribution. The slopes of the resulting straight lines in Figure 1(B) are the molar magnetic susceptibilities, in c.g.s. units, given by $\chi_{\mathrm{D}}=-441(1) \times 10^{-6} \mathrm{~cm}^{3}$ and $\chi_{\mathrm{D}}=-602(4) \times 10^{-6} \mathrm{~cm}^{3}$ per mol Pt atoms of $\mathrm{Pt}_{13} \mathrm{H}_{18}$ and $\mathrm{Pt}_{13} \mathrm{H}_{38}$, respectively. The latter sample is saturated with hydrogen at a pressure of 540 mbar. ${ }^{[11]}$ These values are spectacular, larger by as much as a factor of 37-50 compared with expected values for nonmetallic diamagnetic Pt $\left[\chi_{\mathrm{D}}(\mathrm{Pt}) \approx 2 X_{\mathrm{D}}\left(\mathrm{PtCl}_{2}\right)-\chi_{\mathrm{D}}\left(\mathrm{PtCl}_{4}\right)=-11 \times\right.$ $10^{-6} \mathrm{~cm}^{3} \mathrm{~mol}^{-1}$; or $\chi_{\mathrm{D}}(\mathrm{Pt}) \approx \chi_{\mathrm{D}}\left(\mathrm{PtCl}_{2}\right)-\chi_{\mathrm{D}}\left(\mathrm{Cl}_{2}\right)=-13.5 \times 10^{-6} \mathrm{~cm}^{3}$ $\left.\mathrm{mol}^{-1}\right]{ }^{[16]}$

All Pt species contribute to the diamagnetic susceptibility. The origin of this property is normally attributed to ring currents on atoms which lead to a magnetic moment that is antiparallel to the external magnetic field (Figure 2). It is analogous to the mechanism which is responsible for the large diamagnetic screening in proton NMR of benzene. For a closed-shell cluster consisting of $N$ atoms electron spin paramagnetism and orbital diamagnetism vanish, and the dimensionless volume susceptibility $\chi$ is given to first order in S.I. units by: ${ }^{[17]}$

$$
\chi=-\frac{e^{2} \mu_{0}}{6 m_{e}} \frac{N}{V} \sum_{i}\left\langle r_{i}^{2}\right\rangle,
$$

where $\mu_{0}$ is the vacuum permeability, and $e$ and $m_{\mathrm{e}}$ are the electron charge and mass, respectively. $V$ is the atomic volume, $0.0152 \mathrm{~nm}^{3}$ based on the bulk density of $\mathrm{Pt}$, and $\left\langle r_{i}^{2}\right\rangle$ is the mean square electron distance of the $i$-th electron from the center. To convert eqn. (1) to molar susceptibilities $\chi_{\mathrm{m}}, \chi$ has to be multiplied by the molar volume, $9.09 \mathrm{~cm}^{3} \mathrm{~mol}^{-1}$, and to convert to the more conventional c.g.s. units $\chi_{\mathrm{D}}$ in which all values are quoted here it has to be divided by $4 \pi$. In atoms $\left\langle r_{i}^{2}\right\rangle$ is on the order of $a_{0}^{2}$, where $a_{0}$ is the Bohr radius. The ground state electron configuration of $\mathrm{Pt}$ is [Xe $44 \mathrm{f}^{14} 5 \mathrm{~d}^{9} 6 \mathrm{~s}^{1}$.

In the superatom picture it will be the delocalized cluster molecular orbitals which determine diamagnetism. These provide larger radii for the ring currents (see Figure 2), but it appears nevertheless challenging to explain the experimental enhancement by a factor 37-50, depending on hydrogen coverage. In addition to the conventional atomic values (i) we discuss three possible contributions: (ii) Surprisingly, it was found necessary to involve the shell of chemisorbed hydrogen atoms. The 1 s atomic orbital of $\mathrm{H}$ is energetically significantly lower than the $5 \mathrm{~d}$ of $\mathrm{Pt}$ to which it is bound. The Pt-H bonding orbital will thus also be of low energy,

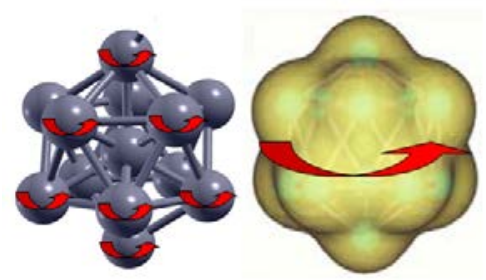

about $5 \mathrm{eV}$ below the Fermi level, making $\mathrm{H}$ partly hydride-like (the antibonding orbital is slightly above the Fermi level ${ }^{[18]}$ ). In DFT quantum chemical calculations the bridge-bonded site along the cluster edge was predicted the most stable, but this is without any corrections for zero-point vibrational motion, and the difference to the on-top Pt and the threefold hole sites is not large. ${ }^{[14,19]}$

Figure 2. The mechanism of diamagnetism is ascribed to magnetic field induced ring currents which are localized on the atoms and lead to a smal magnetic moment that scales with the square of the radius and is antiparallel to the external magnetic field (left). Due to the superatom nature of clusters the ring current operates in the delocalized valence orbitals (right). The strongly enhanced diamagnetism is due to the cluster radius and the large number of involved electrons. 
Table 1. Diamagnetic susceptibility $\chi_{\mathrm{D}}$ of $\mathrm{Pt}_{13} \mathrm{H}_{\mathrm{x}}$ clusters in units of $10^{-6} \mathrm{~cm}^{3}$ per mol Pt atoms

\begin{tabular}{|c|c|c|c|c|c|c|}
\hline \multirow[t]{2}{*}{ sample } & \multicolumn{5}{|c|}{ Calculated contribution (number of electrons) } & \multirow{2}{*}{$\begin{array}{l}\text { Experimental } \\
\text { value }\end{array}$} \\
\hline & (i) $[\mathrm{Xe}] 4 \mathrm{f}^{14}$ core $^{\mathrm{b})}$ & (ii) Hydride shell ${ }^{c}$ & $\begin{array}{l}\text { (iii) } 5 \mathrm{~d} 6 \mathrm{~s} \text { shell } \\
\text { Overall } \mathrm{Pt}_{13} \text { cluster }\end{array}$ & $\begin{array}{l}\text { (iv) } 5 \mathrm{~d} 6 \mathrm{~s} \text { shell } \\
\mathrm{Pt}_{4} \text { tetrahedra }\end{array}$ & sum & \\
\hline $\mathrm{Pt}_{13} \mathrm{H}_{38}$ & -12 & $-313(76 / 13)$ & $-135((120-38) / 13)$ & $-62((40-38 \cdot 3 / 12) / 4)$ & -522 & $-602(4)$ \\
\hline $\mathrm{Pt}_{13} \mathrm{H}_{18}$ & -12 & $-148(36 / 13)$ & $-167((120-18) / 13)$ & $-72((40-18 \cdot 3 / 12) / 4)$ & -399 & $-441(1)$ \\
\hline $\mathrm{Pt}_{13}$ & -12 & 0 & $-200(120 / 13)$ & $-81(40 / 4)$ & -293 & Not measured \\
\hline
\end{tabular}

[a] $105 \mathrm{~d}$ and $6 \mathrm{~s}$ electrons for each of the 12 surface $\mathrm{Pt}$ atoms, diminished by the number of electrons which are involved in the Pt- $\mathrm{H}$ bonds. $\chi_{\mathrm{D}}$ is given per atom of the 13-atom cluster.

[b] Approximate experimental value for $\mathrm{Pt}^{2+}$, see text. The number should be diminished slightly due to the atomic contribution of the remaining

$8 \mathrm{~d}$ electrons which are part of the $5 \mathrm{~d} 6 \mathrm{~s}$ superatom shell.

[c] Two electrons per $\mathrm{Pt}-\mathrm{H}$ bond.

The $\mathrm{H}-\mathrm{H}$ distance of saturated Pt clusters amounts to about $0.22 \mathrm{~nm}$ or $4.7 a_{0}{ }^{[19]}$ At this distance there is considerable overlap of the $\mathrm{H}$ $1 \mathrm{~s}$ electrons, in particular in the hydridic state, which justifies regarding the $\mathrm{Pt}$ bound hydrides as a set of doubly occupied delocalized cluster molecular orbitals. ${ }^{[19]}$ Approximating the radial distance of this shell from the cluster center by the sum of the Pt-Pt and the Pt-H bond distances $(0.277 \mathrm{~nm}+0.158 \mathrm{~nm}=0.435 \mathrm{~nm})$ we arrive at a contribution to $\chi_{\mathrm{D}}$ of $-53.5 \times 10^{-6} \mathrm{~cm}^{3}$ per mol hydride electrons. Per Pt atom this yields $\chi_{\mathrm{D}}=-148 \times 10^{-6} \mathrm{~cm}^{3}$ for $\mathrm{Pt}_{13} \mathrm{H}_{18}$, and $-313 \times 10^{-6} \mathrm{~cm}^{3}$ for $\mathrm{Pt}_{13} \mathrm{H}_{38}$. (iii) The second contribution is due to the delocalized Pt valence electrons. $r_{\mathrm{i}}$ is the positive cluster core radius, which is taken here as the bulk Pt interatomic distance, $d=$ $0.277 \mathrm{~nm} .{ }^{[20]}$ On this basis we obtain $\chi_{\mathrm{D}}=-21.6 \times 10^{-6} \mathrm{~cm}^{3}$ per mol valence electrons. (iv) Induced ring currents cannot only extend over the entire cluster, but also over smaller building blocks. A 13-atom cuboctahedron consists of one central atom surrounded by six atoms in one plane. Three adatoms are found on top and three more below this plane. This structure can be thought of as composed of 6 edgesharing $\mathrm{Pt}_{4}$ tetrahedra, three pointing up from the central plane and three down. The four atoms sit on a sphere with a radius $R=$ $0.25 \cdot 6^{1 / 2} d$, leading to an increment $\chi_{\mathrm{D}}=-8.1 \times 10^{-6} \mathrm{~cm}^{3}$ per mol valence electrons.

There are also $144 \mathrm{f}$ electrons per atom. If they were delocalized over the cluster this would lead to a contribution of the required magnitude. However, they are energetically lower by $73 \mathrm{eV}$ than the $5 \mathrm{~d}$ and 6 s electrons and have an atomic $\left\langle r^{2}\right\rangle$ expectation value of only $0.3 a_{0}{ }^{2}$, an order of magnitude less than the $4 \mathrm{~d}$ electrons. ${ }^{[21]}$ This leaves them little probability of overlap and delocalization, so they will contribute only to the atomic core values. Indeed, they are not normally regarded as valence orbitals.

The four contributions to $\chi_{\mathrm{m}}$ are collected in Table 1 . Contribution (iv) of the $\mathrm{Pt}_{4}$ tetrahedra appears to be minor but nonnegligible. Furthermore, for icosahedra in place of cuboctahedra the building blocks would have to be constructed differently. The added contributions account for $87 \%$ of the experimental value for $\mathrm{Pt}_{13} \mathrm{H}_{38}$ and to $90 \%$ for $\mathrm{Pt}_{13} \mathrm{H}_{18}$. The predicted values are somewhat low, but considering the extensive and debatable approximations in the model the results are still considered to be in perhaps amazing qualitative agreement with experiment. The advantage of the model is that it provides insight into the possible mechanism of diamagnetism in nanoparticles. Detailed quantum chemical predictions would be highly desirable, but they seem currently to be out of range for a heavy element like Pt where relativistic effects are crucial.

The SQUID results represent an ensemble average over all $\mathrm{Pt}$ species, but potential size and shape inhomogeneities are expected to play a limited role for the diamagnetism. The agreement between theoretical model and experimental result suggests that the observed phenomenon has nothing to do with a superconducting transition, even though the clusters have $10^{2}-10^{3}$ delocalized electrons. ${ }^{[4]}$ Since the EPR multiplet confirms that the clusters are non-metallic this excludes also other origins such as a negative Knight shift. Instead, we believe that this is the first experimental observation of the predicted anomalously enhanced diamagnetic susceptibility in a well-defined finite size delocalized system.

A superatom shell-type electronic structure has also been predicted in density functional calculations for $\mathrm{Pt}_{13}$ clusters which did not include any chemisorbed hydrogen atoms. ${ }^{[22]}$ The present essentially quantitative agreement strongly supports that the superatom concept provides a useful description for understanding small spherical clusters. Both, this concept and the strongly enhanced diamagnetism are characteristic and unique for nanomaterials with delocalized electron systems.

It would be interesting to investigate the transition to bulk behavior which has been predicted to occur at a nanoparticle size with a radius on the order of $10 \mu \mathrm{m} .^{[10]}$

Keywords: diamagnetism $\cdot$ Pt clusters · superatoms · EPR

[1] R. König, A. Schindler, T. Herrmannsdörfer, Phys. Rev. Lett. 1999, 82, 4528-4530

[2] A. Schindler, R. König, T. Herrmannsdörfer, H. F. Braun, Phys. Rev. B 2000, 62, 14350-14358.

[3] A. Schindler, R. König, T. Herrmannsdörfer, H. F. Braun, G. Eska, D. Günther, M. Meissner, M. Mertig, R. Wahl, W. Pompe, Physica B 2003, 329-333, 1427-1428.

[4] V. Z. Kresin, Yu. N. Ovchinnikov, Physics Uspekhi, 2008, 51, 427-435. 
[5] Y. Yamamoto, T. Miura, Y. Nakae, T. Teranishi, M. Miyake, H. Hori, Physica (Amsterdam), 2003, 329B-333B, 1183-1184.

[6] X. Liu, M. Bauer, H. Bertagnolli, E. Roduner, J. van Slageren, F. Phillipp, Phys. Rev. Lett., 2006, 97, 253401-1 - 253401-4; and 2009, 102, 049902.

[7] J. Bartolomé, F. Bartolomé, L. M. Garcia, E. Roduner, Y. Akdogan, F. Wilhelm, A. Rogalev, Phys. Rev. B 2009, 80, 014404-1-014404-10.

[8] J. E. Dam, P. C. M. Gubbens, G. J. van den Berg, Physica, 1973, 70, 520546.

[9] A. I. Buzdin, O. V. Dolgov, Yu. E. Lozovik, Phys. Lett. 1984, 100A, 261263.

[10] V. Kresin, Phys. Rev. B 1988, 38, 3741-3746.

[11] C. Jensen, D. Buck, H. Dilger, M. Bauer, F. Phillipp, E. Roduner, Chem. Commun. 2013, 49, 588-590, see also supplementary information.

[12] C. Jensen, J. van Slageren, P. Jakes, R.-A. Eichel, E. Roduner, J. Phys. Chem. B, 2013, 117, 22732-22745.

[13] C. Jensen, PhD thesis, University of Stuttgart, 2013. http://elib.uni-stuttgart.de/opus/volltexte/2013/8361/pdf/Diss_Jensen.pdf [14] X. Liu, H. Dilger, R. A. Eichel, J. Kunstmann, E. Roduner, J. Phys.

[*] Prof. E. Roduner, Dr. C. Jensen, Prof. J. Van Slageren Institute of Physical Chemistry, University of Stuttgart D-70563 Stuttgart (Germany)

E-mail: E.Roduner@ipc.uni-stuttgart.de

Dr. R. A. Rakoczy, Dr. O. Larlus

Clariant Produkte Deutschland $\mathrm{GmbH}$, Lenbachplatz 6 D-80333 Munich, Germany.

Prof. M.Hunger,

Institute of Technical Chemistry, University of Stuttgart

D-70563 Stuttgart (Germany)

Prof. E. Roduner

University of Pretoria, 0002 Pretoria, Republic of South Africa

[**] We thank Hermann Stoll for useful discussions and the $1^{\text {st }}$ Physical Institute of the University of Stuttgart for letting us use their SQUID magnetometer.
Chem. B, 2006, 110, 2013-2023.

[15] U. Rohrmann, R. Schäfer, Phys. Rev. Lett., 2013, 111, 133401-1 133401-5

[16] CRC Handbook of Chemistry and Physics, $55^{\text {th }}$ Edition (CRC Press, Cleveland, OH, 1974-75).

[17] S. Blundell, Magnetism in Condensed Matter, Oxford Masters Series in Condensed Matter Physics (Oxford University Press, Oxford, 2001).

[18] B. Hammer, J. K. Nørskov, Nature, 1995, 376, 238-240.

[19] L. Chen, Ch. Zhou, J. Wu, H. Cheng, Front. Phys. China 2009, 4, 356366.

[20] N. Watari, S. Ohnishi, Phys. Rev. B 1998, 58, 1655-1677.

[21] J. P. Desclaux, At. Data Nucl. Data Tables 1973, 12, 311-406.

[22] N. Watari, S. Ohnishi, J. Chem. Phys. 1997, 106, 7531-7540. 


\section{Supporting Information}

Materials and Methods: The LTL-type material used for this study was synthesized by using iron-free tetraethoxysilane TEOS (99\%, Fluka) and aluminum isopropoxide $\mathrm{Al}{ }^{\mathrm{i}} \mathrm{PrO}(98 \%$, Fluka) as silica and alumina raw chemicals, but also potassium hydroxide and distilled water. The precursor gel was prepared by dissolving in a polypropylene bottle the potassium hydroxide in water, followed by the addition of the $\mathrm{Al}$ PrO alumina source. This suspension was mixed at room temperature about two hours, until a clear solution was observed, indicating that the aluminum species were completely dissolved in water. The TEOS silica source was then added to the solution and stirred at room temperature in order to ensure a homogeneous hydrolysis of the alkoxy-silica species in the solution, typically for half an hour. The solution was stirred for two additional hours at room temperature and then aged overnight without stirring at $20^{\circ} \mathrm{C}$. The polypropylene bottle was kept sealed during the entire preparation of the precursor gel, preventing the evaporation of the isopropanol and ethanol resulting from the hydrolysis of the TEOS and $\mathrm{Al}^{\mathrm{i}} \mathrm{PrO}$ reactants. The precursor gel had the following molar composition: $1.00 \mathrm{SiO}_{2}: 0.10 \mathrm{Al}_{2} \mathrm{O}_{3}: 0.30 \mathrm{~K}_{2} \mathrm{O}: 20$ $\mathrm{H}_{2} \mathrm{O}$. After having been poured in the autoclave, the gel was submitted to a hydrothermal treatment at $150^{\circ} \mathrm{C}$ for 72 hours. At the end of the synthesis, the autoclave was quenched down to room temperature and the product was collected by filtration and washed with distilled water until a $\mathrm{pH}$ of about 9.0 was measured. Thereafter the product was dried at $120^{\circ} \mathrm{C}$ overnight and was then ready for the investigation. The $\mathrm{X}$ ray powder diffraction pattern exhibits all the features of a pure LTL material (Figure S1). No extra peak that can be assigned to a side phase can be seen. The large peak widths mean that the LTL crystal size is extremely small, most probably close to the nano-sized domain. Elemental analysis gave an $\mathrm{Si} / \mathrm{Al}$ ratio of 2.8 .

Characterization: MAS NMR experiments of the iron-free KL zeolite were performed on a BRUKER Avence III 400WB spectrometer. In the ${ }^{27} \mathrm{Al}$ MAS NMR (MAS: magic angle spinning) spectrum, a single line at $59.3 \mathrm{ppm}$ caused by tetrahedrally coordinated framework aluminum atoms was observed (Fig. S2A). Signals at $-91.2,-96.8,-101.5$, and $-106.5 \mathrm{ppm}$ due to $\mathrm{Si}(1 \mathrm{Si}, 3 \mathrm{Al}), \mathrm{Si}(2 \mathrm{Si}, 2 \mathrm{Al}), \mathrm{Si}(3 \mathrm{Si}, 1 \mathrm{Al})$, and $\mathrm{Si}(4 \mathrm{Si})$ species in the LTL framework, respectively, were found in the ${ }^{29} \mathrm{Si}$ HPDEC MAS NMR (HPDEC: high-power proton decoupling) spectrum (Fig. S2B). The quantitative evaluation of the relative intensities in the latter spectrum gave the ratio $n_{\mathrm{Si}} / n_{\mathrm{Al}}=2.3$ for framework T atoms, in satisfactory agreement with elemental analysis. The crystalline phase is intact and no extra-framework aluminum is observed.

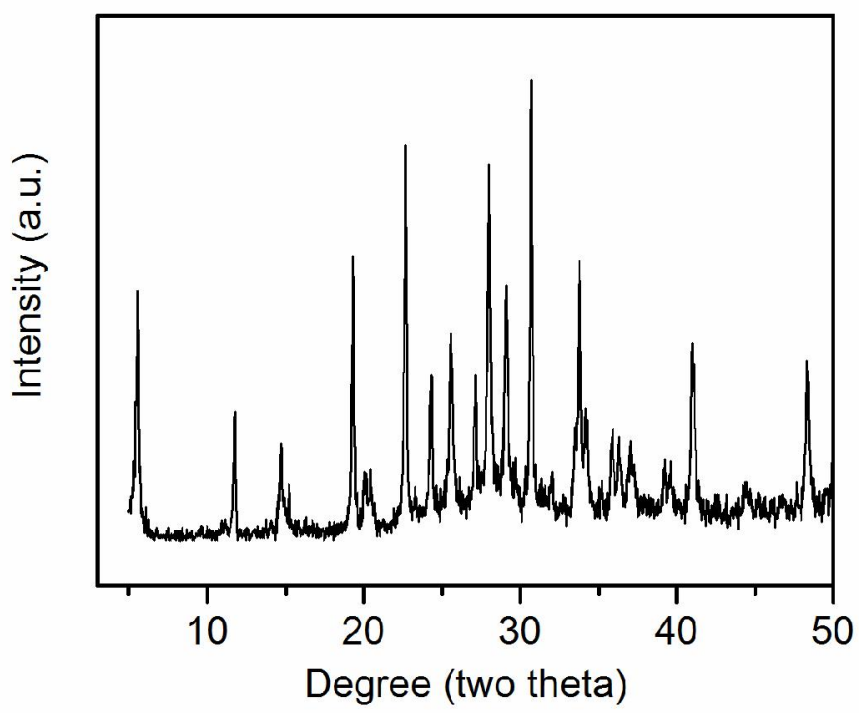

Figure S1. X-ray powder diffraction pattern of iron-free LTL zeolite. 

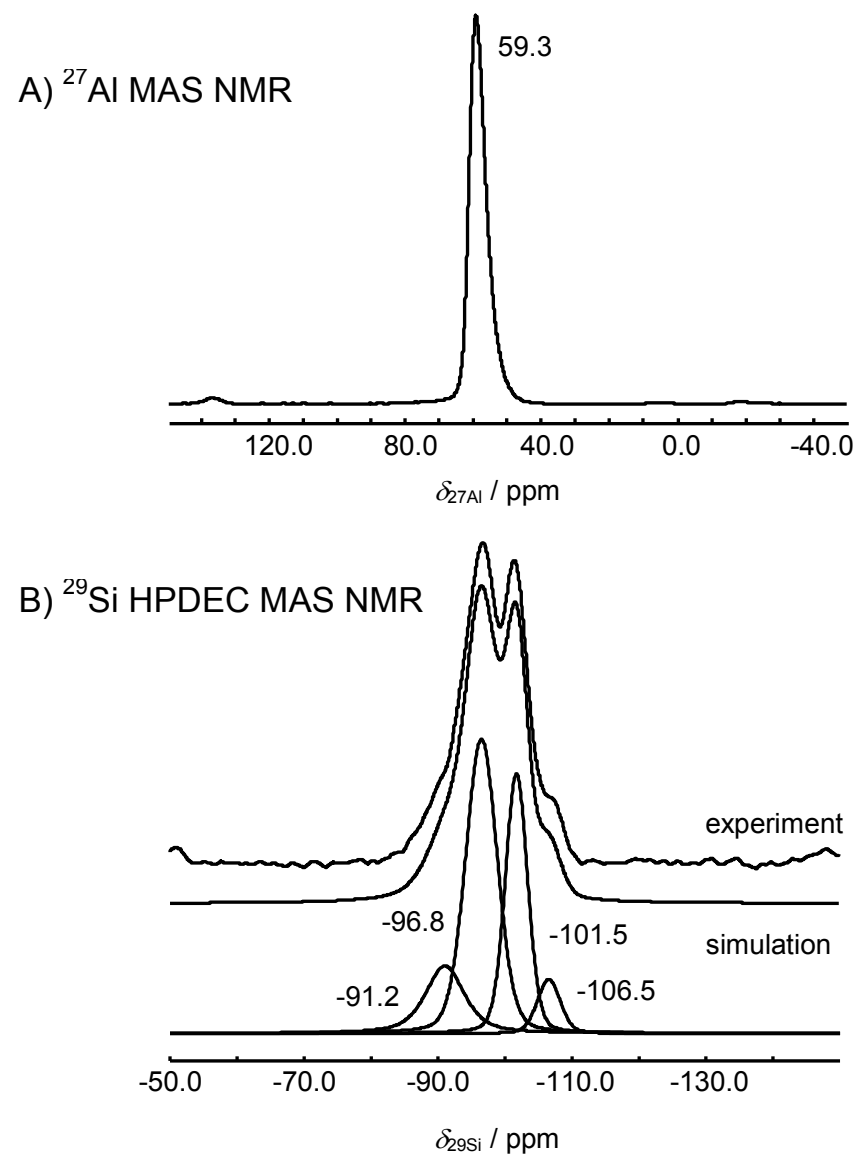

Figure S2. ${ }^{27} \mathrm{Al}$ MAS NMR (A) and ${ }^{29}$ Si HPDEC MAS NMR (B) spectra of iron-free zeolite K-LTL.

The EPR spectrum of a $\mathrm{Pt}_{13} \mathrm{D}_{\mathrm{X}}$ cluster shows a multiplet spectrum with a regular splitting (see Figure S3). The pattern fits well the hyperfine interaction of the unpaired electron with 12 equivalent $\mathrm{Pt}$ nuclei in natural isotopic abundance. The spin density on the $13^{\text {th }}$ atom in the cluster center is small. This is understood on the basis that this highly symmetric cluster should be regarded as a superatom where the cluster molecular orbital containing the unpaired electron resembles an atomic orbital with $\ell>0$ that has a node in the center, just as in normal atoms. Further splitting by the chemisorbed hydrogen is not resolved but contributes to the line width. The EPR signal relates to an electron $g$ value of 2.3675 , reflecting a significant spin-orbit coupling as expected for transition metals with the unpaired electron in a more than halffilled d-orbital. The resolved multiplet reflects the nature of the clusters, which is molecular rather than metallic. The latter should give a single line due to frequent nuclear spin flips induced by scattering with conduction electrons.

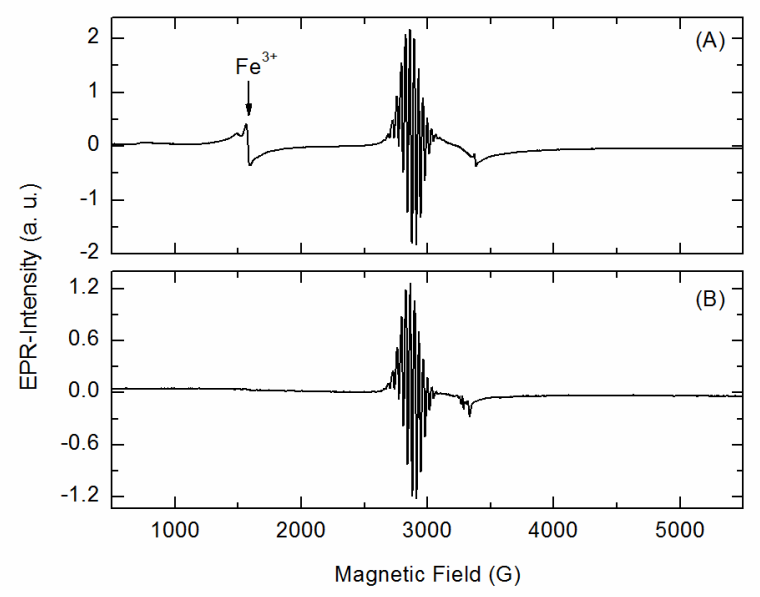

Figure S3. EPR spectrum of $\mathrm{Pt}_{13} \mathrm{D}_{\mathrm{X}}$ measured at $4 \mathrm{~K}$ of $5.3 \mathrm{wt} . \% \mathrm{Pt}$ supported on a commercial KL-zeolite (A) and of $5.5 \mathrm{wt} . \% \mathrm{Pt}$ on a specially prepared iron-free KL-zeolite that was used for the present work (B). 


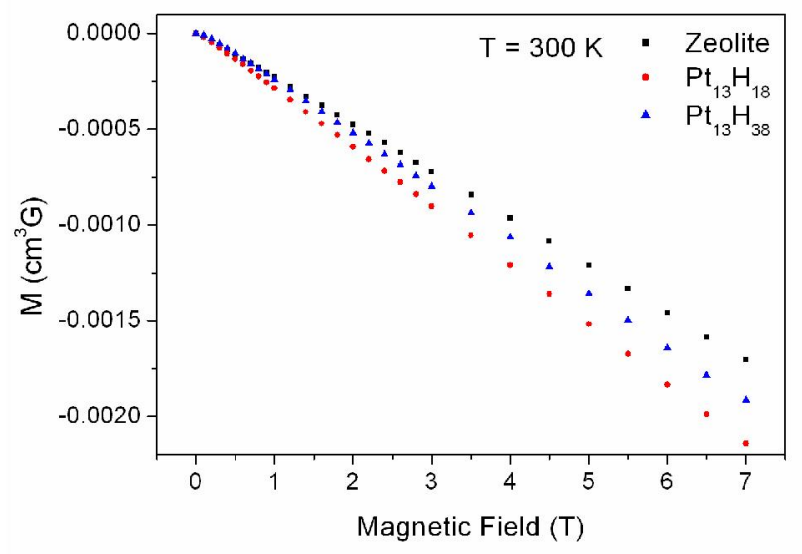

Figure S4. Raw data of the SQUID magnetization measurements for three samples: Pt-free zeolite with a mass $\mathrm{m}_{\text {zeolite }}$ of $63.5 \mathrm{mg}$, zeolite with $5.5 \mathrm{wt} \% \mathrm{Pt}$ in the form of $\mathrm{Pt}_{13} \mathrm{H}_{18}$ clusters $\left(\mathrm{m}_{\mathrm{Pt} 13 \mathrm{H} 18}=63.0 \mathrm{mg}, \mathrm{n}_{\mathrm{Pt}}=17.76 \mu \mathrm{mol}\right)$ and zeolite with $5.5 \mathrm{wt} \% \mathrm{Pt}_{\text {in }}$ the form of $\mathrm{Pt}_{13} \mathrm{H}_{38}$ clusters $\left(\mathrm{m}_{\mathrm{Pt} 13 \mathrm{H} 38}=52 \mathrm{mg}, \mathrm{n}_{\mathrm{Pt}}=14.66 \mu \mathrm{mol}\right)$. Subtraction of the magnetization of the empty zeolite, $\mathrm{M}_{\text {zeolite }}$, from the magnetization of the Pt-loaded zeolites, $\mathrm{M}_{\mathrm{Pt}-z e o l i t e}$, and normalization to obtain the molar magnetizations of $\mathrm{Pt}$ as displayed in Figure 1(B) was done using the following formula:

$$
M_{P t}=\left(\frac{M_{P t-\text { zeolite }}-\frac{M_{\text {zeolite }} \cdot 0.945 \cdot m_{\text {Pt-zeolite }}}{m_{\text {zeolite }}}}{n_{P t}}\right)
$$

The difference in the numerator, i.e. the magnetization due to the small amount of Pt alone, amounts to 25-30\% of the magnetization of the

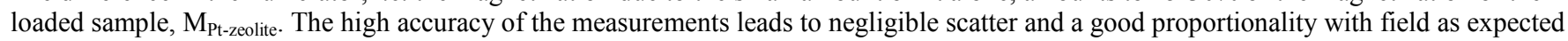
for diamagnetism (see Figure 1(B)). 